\title{
Silver nanoparticles: synthesis and application in mineralization of pesticides using membrane support
}

\author{
G. Manimegalai $\cdot$ S. Shanthakumar • \\ Chandan Sharma
}

Received: 17 December 2013/Accepted: 14 May 2014/Published online: 27 May 2014

(C) The Author(s) 2014. This article is published with open access at Springerlink.com

\begin{abstract}
Pesticides are deliberately used for controlling the pests in agriculture and public health, due to which, a part of it is present in the drinking water. Due to their widespread use, they are present in both surface and ground water. Most of the pesticides are resistant to biodegradation and are found to be carcinogenic in nature even at trace levels. Conventional methods of pesticide removal are disadvantageous due to their inherent time consumption or expensiveness. Nanoparticles alleviate both of these drawbacks and hence, they can be effectively utilized for the mineralization of pesticides. To prevent the presence of nanoparticles in the purified water after mineralization of pesticides, they need to be incorporated on a support. In earlier studies, researchers employed activated carbon and alumina as support for silver nanoparticles in pesticide mineralization. However, not many studies have been carried out on polymeric membranes as support for silver nanoparticles in the mineralization of pesticides (chlorpyrifos and malathion). With this in view, a detailed study has been carried out to estimate the mineralization potential of silver nanoparticles (synthesized using glucose) supported on cellulose acetate membrane. It is observed that the silver nanoparticles can effectively mineralize the
\end{abstract}

\footnotetext{
G. Manimegalai $\cdot$ S. Shanthakumar

Environmental Engineering Division, School of Mechanical and

Building Sciences, VIT University, Vellore 632014, India

e-mail: maniganesh87@gmail.com

S. Shanthakumar

e-mail: sskumariit@gmail.com

C. Sharma $(\square)$

Chemical Engineering Division, School of Mechanical and Building Sciences, VIT University, Vellore 632014, India

e-mail: chandan1816@gmail.com
}

pesticides, and the concentration of nanoparticles enhances the rate of mineralization.

Keywords Pesticide - Nanoparticles $\cdot$ Cellulose acetate membrane $\cdot$ Mineralization $\cdot$ Chlorpyrifos $\cdot$ Malathion

\section{Introduction}

Pesticides hold a unique position among contaminants found in water, since they are deliberately used to control pest in agriculture and public health [1]. Water pollution due to pesticides is a critical problem in developing countries. The sources of pesticides in water are runoff from agricultural fields, industrial wastes and orchards treated with pesticides [2]. They comprise different classes such as insecticides, fungicides, herbicides and rodenticides etc. Due to their lack of high specificity, pesticides are toxic to many unintended species including humans.

Because of their widespread use, they leach into surface and ground water and are therefore present in drinking water as well. They persist in the environment and pose a significant health threat especially to humans. Among the possible effects related to this exposure, genetic damage has important health implications for the induction of lung cancer, non-Hodgkin's lymphoma, pancreatic cancer, bladder cancer and leukemia [3, 4].

Due to the increased awareness about the risks associated with drinking water contamination, the allowable limits are being revised and the permissible limits are expected to reach molecular levels in the coming years. Despite the negative perception of the public, pesticides are still going to be used for many decades to ensure the food supply for the ever-growing world population [5]. 
Therefore, it becomes essential to develop new technologies which are capable of removing pesticides even at trace levels.

Earlier methods of pesticide removal include photocatalysis [6-9], biodegradation [10, 11], adsorption and membrane separation [2]. These methods are disadvantageous either due to their time consumption or expensiveness. The impacts of nanotechnology are increasingly evident in all areas of science and technology, including the field of environmental studies and treatment $[12,13]$. Nanoparticles are usually referred to as clusters of atoms in the size range of 1-100 $\mathrm{nm}$ [14].

Nanoparticles exhibit a completely new or improved properties compared to larger particles of bulk material and these novel properties are derived due to the variation in specific characteristics such as size, ionic state, distribution and morphology of the particles. Nanoparticles exhibit a higher surface area-to-volume ratio with a decrease in the size of particles [15]. It has been well established that the metallic nanoparticles such as zerovalent iron, copper, silver and gold have unique catalytic activity in the mineralization of halocarbons and other organic as well as inorganic contaminants. Membrane plays a pivotal part in water treatment technology as there is a huge demand for low energy consumption processes [16]. In addition, the membrane technology used as a support for the nanoparticles' dispersion has great energy advantages, furthermore it decreases the chemical usage for membrane cleaning and fabrication cost, as compared to other conventional methods [1719]. However, not many studies have been carried out on cellulose acetate membrane (CAM) as support for silver nanoparticles (AgNPs) in the mineralization of pesticides in water. With this in view, a detailed study has been carried out to estimate the mineralization potential of silver nanoparticles supported on CAM, and the critical evaluation of the results is presented in this paper.

\section{Materials and methods}

\section{Chemicals and reagents}

Silver nitrate, glucose, diethyl amine, cellulose acetate, hexane and acetone were purchased from Thomas baker, India. All the reagents used were of analytical reagent grade and were used as received. Milli-Q water was used for the synthesis of silver nanoparticles and double-distilled water was used for the mineralization of pesticides. Commercially available pesticides such as chlorpyrifos (Fig. 1a) and malathion (Fig. 1b) were obtained from local pesticide shop.
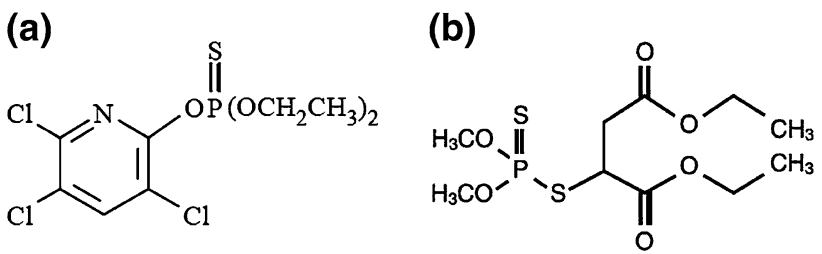

Fig. 1 Structure of a chlorpyrifos and $\mathbf{b}$ malathion

Synthesis of silver nanoparticles using glucose as reducing agent

To obtain a homogenous solution, $25 \mathrm{~mL}$ of $100 \mathrm{mM}$ $\mathrm{AgNO}_{3}$ and $25 \mathrm{~mL}$ of $50 \mathrm{mM}$ glucose were mixed together and stirred. $25 \mathrm{~mL}$ of $115 \mathrm{mM}$ diethylamine was added to the above solution and stirred vigorously. The color of the solution was changed to black and then brown. Finally, a light green colored precipitate was formed. After decanting and washing repeatedly three times with distilled water, the precipitate was collected and dried in oven at $50-60{ }^{\circ} \mathrm{C}[20]$.

Cellulose acetate membrane preparation incorporated with AgNPs

Each membrane was prepared using $50 \mathrm{~mL}$ of $12 \mathrm{wt} \%$ cellulose acetate dissolved in acetone. After the complete dissolution, required quantity of AgNPs was added and kept in the magnetic stirrer to obtain homogenous solution. The homogenous viscous solution thus obtained was casted on a clean and dry glass plate using a doctor blade (gap $0.625 \mathrm{~mm}$ ). The film was air dried for $30 \mathrm{~s}$. Further, it was immersed in ethanol for $20 \mathrm{~min}$ and taken out for the mineralization process [21].

\section{Extraction of pesticide using hexane}

After the treatment of various concentrations of pesticide solution with AgNPs, $75 \mathrm{~mL}$ of hexane was added to $500 \mathrm{~mL}$ of the treated water. It was mixed well and kept for $30 \mathrm{~min}$ for the separation into layers of hexane with pesticide and water. The hexane with the extracted pesticide was kept out for evaporation of hexane till the final volume was up to 3-4 mL. It was made up to $10 \mathrm{~mL}$ with hexane and used to find out the absorbance value using UV-Visible spectroscopy [22].

\section{Mineralization of pesticide using CAM incorporated with AgNPs}

Required concentrations of pesticide solutions (1,2 and $3 \mathrm{mg} / \mathrm{L}$ ) were prepared. Cellulose acetate membrane incorporated with various AgNPs concentration (100, 200, 300,400 and $500 \mathrm{mg}$ ) was used for every $250 \mathrm{~mL}$ of pesticide solution. The membrane was dipped into the 
Fig. 2 AFM images of AgNPs, a $5 \times 5 \mu^{2}$ and

b $770 \times 770 \mathrm{~nm}^{2}$ (a)

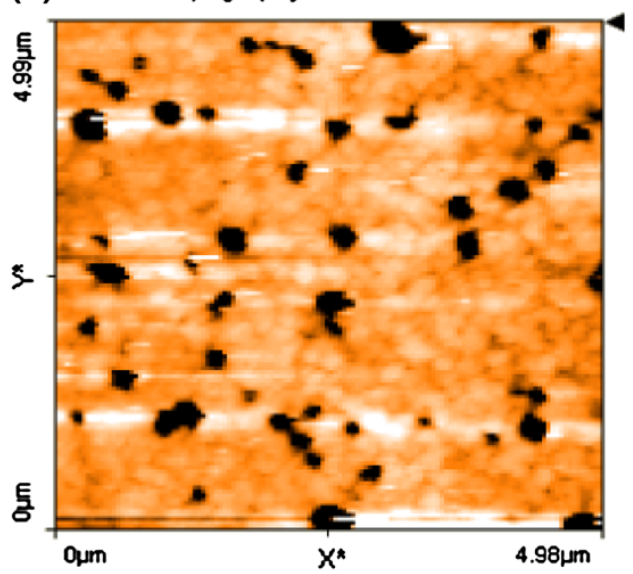

(b) Topography - Scan forward

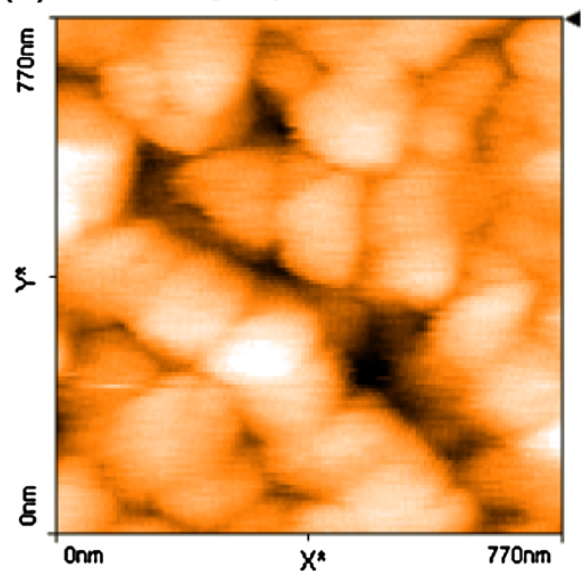

pesticide solution and at every 20 min interval the concentration of pesticides was examined.

\section{Pesticide estimation}

The estimation of chlorpyrifos and malathion was done by measuring the absorbance peak at 292 and $267 \mathrm{~nm}$, respectively [23]. $3.5 \mathrm{~mL}$ aliquots of the solution were taken out at equal time intervals (20 $\mathrm{min}$ ) for analysis using UV-Visible spectroscopy and the solutions were poured back after measuring the absorbance.

\section{Results and discussion}

\section{AFM analysis}

To determine the topological nature of synthesized silver nanoparticles, the sample was coated as a thin film on glass slide for Atomic Force Microscopy (AFM) analysis and the surface was scanned in an area of $5 \times 5 \mu \mathrm{m}^{2}$. Figure 2 depicts the AFM image of silver nanoparticles at different magnifications. It can be observed from the figure that the brighter field represents the silver nanoparticles due to its high modulus as compared to the modulus of cellulose acetate. Further, it can be noted that the silver nanoparticles are spherical in nature and are of uniform size. The size of the silver nanoparticles was measured using ImageJ software and was found in the range of $60-75 \mathrm{~nm}$.

Mineralization of chlorpyrifos using CAM incorporated with AgNPs

It is well known that both chlorpyrifos and malathion belong to organophosphorothioate pesticides. On

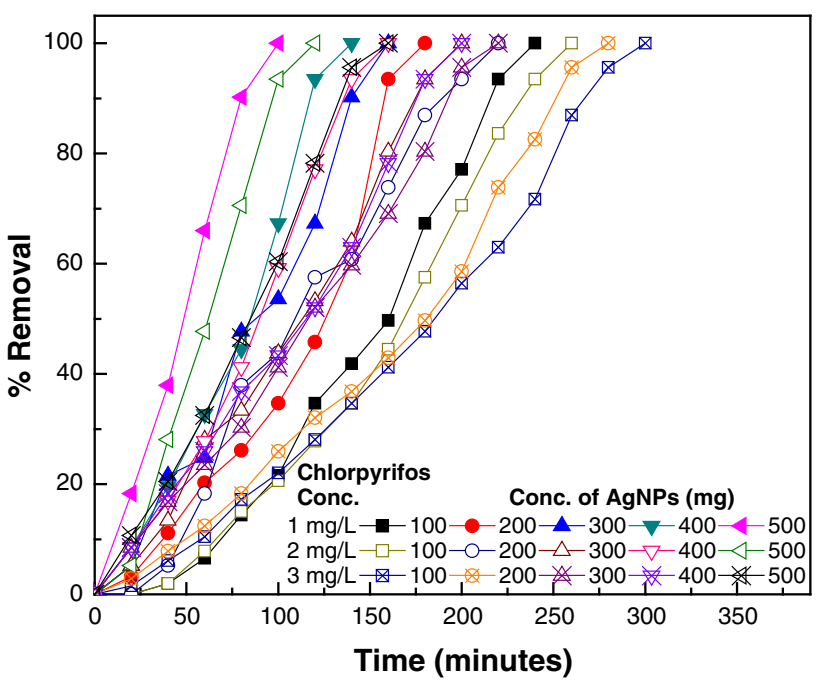

Fig. 3 Mineralization of chlorpyrifos at different AgNPs concentration

interaction of these pesticides with AgNPs, pesticide undergoes mineralization during which the metal changes into metal salt [23]. Figure 3 depicts the time-dependent mineralization process of chlorpyrifos at different concentrations of silver nanoparticles. The rate of mineralization was found to be increasing with AgNPs concentration. This is due to the fact that, degradation of chlorpyrifos takes place with the formation of AgNP-S surface complex therefore, with increase in concentration of nanoparticles in the support, active surface area was found to be increasing and hence, at different AgNPs concentrations, the decrease in time for mineralization process was observed. These observations are consistent with the results reported in the literature. The detailed degradation pathway of chlorpyrifos is mentioned elsewhere [24]. 


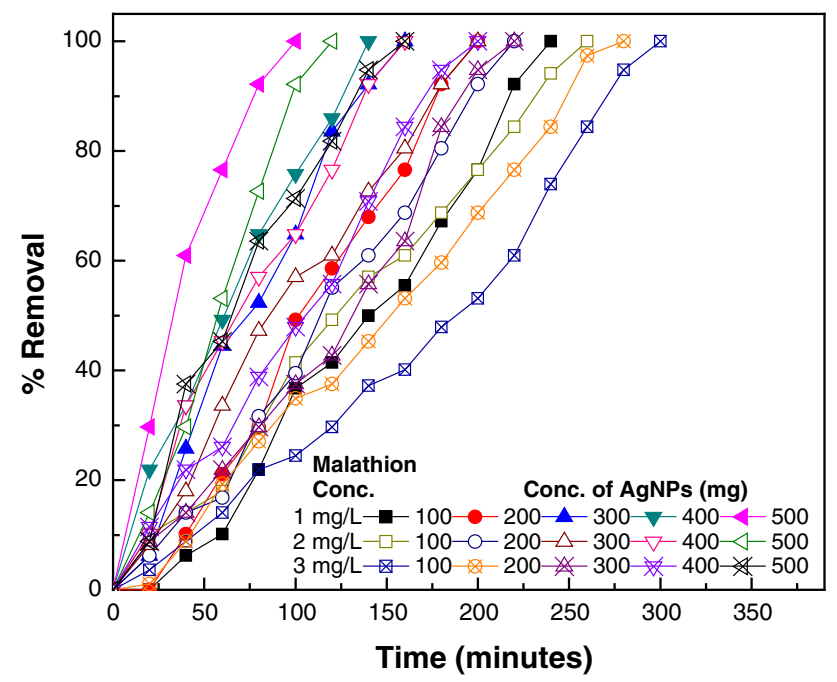

Fig. 4 Mineralization of malathion at different AgNPs concentration

Mineralization of malathion using CAM incorporated with AgNPs

Figure 4 depicts the time-dependent mineralization process of malathion at different concentrations of silver nanoparticles. For a particular concentration of malathion, the time taken for the mineralization process decreases, as the AgNPs concentration increases. This is because as the nanoparticle concentration is increasing, the number of active sites is also increasing and hence, rate of mineralization becomes faster. So, the decrease in time for mineralization process at different AgNPs concentrations was observed.

Complete mineralization of pesticides was observed, this is because on degradation of pesticides the oxidation state of silver remains unaltered. To measure the effectiveness of nanoparticles, time taken for complete mineralization of pesticides at different concentrations of AgNPs was compared. However, for the sake of brevity, the results of the pesticides for $2 \mathrm{mg} / \mathrm{L}$ concentration, only, are presented in the Fig. 5. It can be observed from the figures that for both chlorpyrifos and malathion, the time consumption pattern was comparatively the same.

\section{Conclusion}

Silver nanoparticles are proved to be novel, highly efficient in removing the pesticides. And the method of preparation of AgNPs is found to be economical. Silver nanoparticles were synthesized using glucose as a reducing agent and incorporated on cellulose acetate membrane. The silver nanoparticles' concentration and pesticide concentration influence the mineralization time. Time required for mineralization of both chlorpyrifos and malathion having
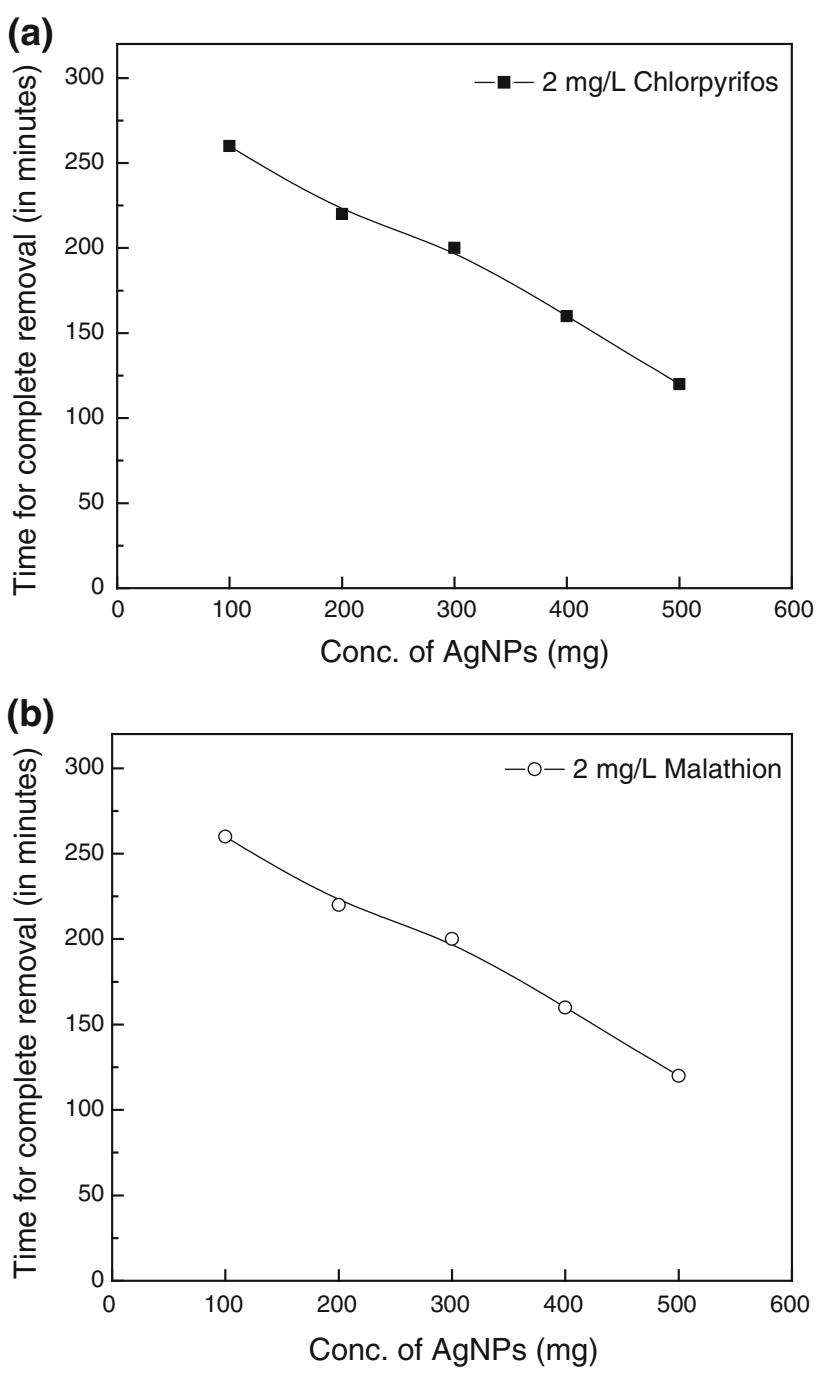

Fig. 5 Time required for complete removal of a chlorpyrifos and b malathion at different concentrations of AgNPs

concentration of $3 \mathrm{mg} / \mathrm{L}$ was observed as 300 and $160 \mathrm{~min}$ when 100 and $500 \mathrm{mg}$ of AgNPs incorporated on cellulose acetate membrane, respectively. Hence, it can be concluded that silver nanoparticles do not discriminate different pesticides and can be effectively used for the removal of pesticides in the rural areas where the pesticide contamination is prevalent.

Acknowledgments Authors are thankful to VIT University for providing all the necessary facilities.

Conflict of interest The authors declare that they have no competing interests.

Author contribution GM was involved in the acquisition and analysis of data. SS was involved in designing the experimental work and drafting the manuscript. CS revised the manuscript critically and gave final approval for submission. All authors read and approved the final manuscript. 
Open Access This article is distributed under the terms of the Creative Commons Attribution License which permits any use, distribution, and reproduction in any medium, provided the original author(s) and the source are credited.

\section{References}

1. Brown, A.W.A.: Ecology of pesticides. Wiley, New York (1997)

2. Savage, N., Duncan, M.D.J., Street, A., Sustich, R.: Nanotechnology applications for clean water. William Andrew Inc, Burlington (2009)

3. Ito, N., Hagiwara, A., Tamano, S., Futacuchi, M., Imaida, K., Shirai, T.: Effects of pesticide mixtures at the acceptable daily intake levels on rat carcinogenesis. Food Chem. Toxicol. 34, 1091-1096 (1996)

4. Sailaja, N., Chandrasekhar, M., Rekhadevi, P.V., Mahbooba, M., Rahmana, M.F., Vuyyuri, S.B., Danadevi, K., Hussain, S.A., Grover, P.: Genotoxic evaluation of workers employed in pesticide production. Mutat. Res. 609, 74-80 (2006)

5. Wang, C.J., Liu, Z.Q.: Foliar uptake of pesticides-present status and future challenge. Pestic. Biochem. Physiol. 87, 1-8 (2007)

6. Herrmann, J.M., Guillard, C.: Photocatalytic degradation of pesticides in agricultural used waters. C R Acad. Sci. II C 3(6), 417-422 (2000)

7. Devipriya, S., Yesodharan, S.: Photocatalytic degradation of pesticide contaminants in water. Sol. Energy Mater. Sol. Cells 86, 309-348 (2003)

8. Shankar, M.V., Cheralathan, K.K., Arabindoo, B., Palanichamy, M., Murugesan, V.: Enhanced photocatalytic activity for the destruction of monocrotophos pesticide by $\mathrm{TiO}_{2} / \mathrm{H} \beta$. J. Mol. Catal. A Chem. 223, 195-200 (2004)

9. Oller, I., Gernjak, W., Maldonado, M.I., Ṕerez-Estrada, L.A., Śanchez-Ṕerez, J.A., Malato, S.: Solar photocatalytic degradation of some hazardous water-soluble pesticides at pilot-plant scale. J. Hazard. Mater. 38, 507-517 (2006)

10. Rodriguez-Cruz, M.S., Jones, J.E., Bending, G.D.: Field-scale study of the variability in pesticide biodegradation with soil depth and its relationship with soil characteristics. Soil Biol. Biochem. 38, 2910-2918 (2006)

11. Rodrıuez-Cruz, M.S., Balum, J., Shaw, L.J., Sorensen, S.R., Shi, S., Aspray, T., Jacobsen, C.S., Bending, G.D.: Biodegradation of the herbicide mecoprop-p with soil depth and its relationship with class III tfdAgenes. Soil Biol. Biochem. 42, 32-39 (2010)

12. Watlington, K.: Emerging Nanotechnologies for Site Remediation and Wastewater Treatment. USEPA Report, Available at:
http://nepis.epa.gov/Adobe/PDF/P1003FG4.PDF. Accessed 8 July 2011

13. Manimegalai, G., Shanthakumar, S., Sharma, C.: Pesticide mineralization in water using silver nanoparticles: a review. Int. J. Chem. Sci. 9, 1463-1471 (2012)

14. Abou, E.N.K.M., Eftaiha, A., Al-Warthan, A., Ammar, R.A.A.: Synthesis and applications of silver nanoparticles. Arab. J. Chem. 3, 135-140 (2009)

15. Gurunathana, S., Kalishwaralala, K., Vaidyanathana, R., Deepaka, V., Pandiana, S.R., Muniyandia, J., Hariharana, N., Eomb, S.H.: Biosynthesis, purification and characterization of silver nanoparticles using Escherichia coli. Colloids Surf. B 74, 328-335 (2009)

16. Kasher, R.: Membrane-based water treatment technologies: recent achievements, and new challenges for a chemist. Bull. Isr. Chem. Soc. 24, 10-18 (2009)

17. Muller, N., Crawley, T.: Nanoenhanced Membranes for improved water treatment. Available at: http://www.observatorynano.eu/ project/filesystem/files/Briefing\%20No.16\%20Nanoenhanced\% 20membranes\%20for\%20water\%20treatment.pdf. Assessed 3 July 2012

18. Balamurugan, R., Sundarrajan, S., Ramakrishna, S.: Recent trends in nanofibrous membranes and their suitability for air and water filtrations. Membranes 1, 232-248 (2011)

19. Tepliakov, V.V.: Impact of membranes and membrane processes on environmental issues. In: Proceedings of the 1st international conference on methods and materials for separation process, pp. 35-36. Wroclaw University of Technology, Poland (2011)

20. Janardhanan, R., Karuppaiah, M., Hebalkar, N., Rao, T.N.: Synthesis and surface chemistry of nano silver particles. Polyhedron 28, 2522-2530 (2009)

21. Wu, L., Shamsuzzoha, M., Ritchiel, S.M.C.: Preparation of cellulose acetate supported zero-valent iron nanoparticles for the dechlorination of trichloroethylene in water. J. Nanopart. Res. 7, 469-476 (2005)

22. Nair, S., Pradeep, T.: Extraction of chlorpyrifos and malathion from water by metal nanoparticles. J. Nanosci. Nanotechnol. 7, 1871-1877 (2007)

23. Pradeep, T., Anushup: Detection and extraction of pesticides from drinking water using nanotechnologies. In: Savage, et al. (ed.) Nanotechnology applications for clean water, pp. 191-212. William Andrew Inc., New York (2009)

24. Bootharaju, M.S., Pradeep, T.: Understanding the degradation pathway of the pesticide, chlorpyrifos by noble metal nanoparticles. Langmuir 28, 2671-2679 (2012) 